DOI: $10.1515 /$ awutp -2015-0101

\title{
NEURAL FLOWS IN HOPFIELD NETWORK APPROACH
}

\author{
Carmen Ionescu, Emilian Panaitescu and Mihai Stoicescu \\ Faculty of Exact Sciences, Department of Physics, University of Craiova, Str. A.I. Cuza, nr.13, 200585, \\ Craiova, Dolj, Romania
}

\author{
Article Info \\ Received: 08.02.2013 \\ Accepted: 27.02.2013 \\ Keywords: neural cell, \\ nonlinear dynamics \\ PACS: $87.85 . d q, 05.45 .-\mathrm{a}$
}

\begin{abstract}
In most of the applications involving neural networks, the main problem consists in finding an optimal procedure to reduce the real neuron to simpler models which still express the biological complexity but allow highlighting the main characteristics of the system. We effectively investigate a simple reduction procedure which leads from complex models of Hodgkin-Huxley type to very convenient binary models of Hopfield type. The reduction will allow to describe the neuron interconnections in a quite large network and to obtain information concerning its symmetry and stability. Both cases, on homogeneous voltage across the membrane and inhomogeneous voltage along the axon will be tackled out. Few numerical simulations of the neural flow based on the cableequation will be also presented.
\end{abstract}

\section{Introduction}

One of the most successful model explaining the functioning of the memory as an asynchronous neural network was proposed in 1982 by John Hopfield [1]. The network consists of $n$ totally coupled units, that is, each unit is connected to all other units except itself and the units change information recursively between them. It is assumed that the individual units are randomly updated, preserving their individual states in the interval between two updates. Specific for the model is that no synchronization requirements are imposed, that is not a universal time is needed. The neurons are not updated simultaneously, but at different moments in a random order. Another supposition in the Hopfield model is that there is a symmetric transfer of information among units, the interaction weight for the transfer from the neuron $i$ to the neuron $j$ being the same as for the transfer in the opposite sense, $w_{i j}=w_{j i}$.

Intensive studies were devoted to this model, either in neurosciences for describing a bidirectional associative memory close with the human memory [2-3], or in artificial intelligence, as an important tool in programming or solving optimization problems [4-5]. The present paper belongs to the first type of approaches mentioned before and it has the following structure: after this introductive section, the second section will present basic facts on the Hopfield network seen as a nonlinear network of interacting neurons. It will be a 
monographic approach pointing out the main characteristics of the model, including its symmetry and strong stability. The third section will present, as a limit case of the general theory, the propagation of the neural flow along an isolated neuron seen as a free subsystem of a Hopfield neural network. The results will be compared with what is already known on the neural flow through an individual neuron. Some concluding remarks will end the paper.

\section{Neural networks with binary states}

The Hopfield network consists in a set of $n$ interconnected neurons, all of them being active both in sending and in receiving information. The neurons could have binary states and the passage from one state to the other is the result of an activation process. For each neuron, the activation is produced randomly, asynchronously and independently of other neurons. The two activation values which define the two possible states of each neuron are usually chosen as +1 and -1 . The model is somehow an extreme simplification of the models trying to capture the biological complexity of the neural cells, as for example Hodgkin-Huxley one [6]. The main idea of these models is that the neuron membrane behaves as a capacitor with many conducting channels joining its two sides, the extracellular and the cytoplasmic ones. Different types of ions existing around could pass through the channels, either because of the electromagnetic forces (electric current), or because of the diffusion process due to the gradient of ion concentration (diffusive current). Moreover, different physical and chemical interactions existing inside the channel can generate supplementary currents, as a genuine current source. The electric circuit that fits best with these assumptions and is usually used for describing the dynamics of neurons is a capacitor in parallel with some linear and nonlinear conductors and with some supplementary ion pumps [7].

We take into consideration, as in the Hodgkin-Huxley model, that the main membrane currents are $I_{N a}, I_{K}$ generated by the sodium and potassium ions. The conduction of all the other ions is represented by the current $I_{L}$ seen as a supplementary ohmic leakage contribution. The conduction take place through voltage dependent channels with the resistance per unit area denoted by $R_{N a}, R_{K}, R_{L}$, respectively. $C_{m}$ represents the membrane capacitance per unit area. The equivalent electric circuit looks as in Fig.1, where $I_{m}$ denotes the synaptic and ion pump currents. In the Hodgkin-Huxley model the resistances, or more precisely the conductances $g=1 / R$ of the $N a$ and respectively $K$ channels have rather complicated dependencies on the instant voltage $V$ on the membrane, while $g_{L}$ is constant (ohmic resistance). 


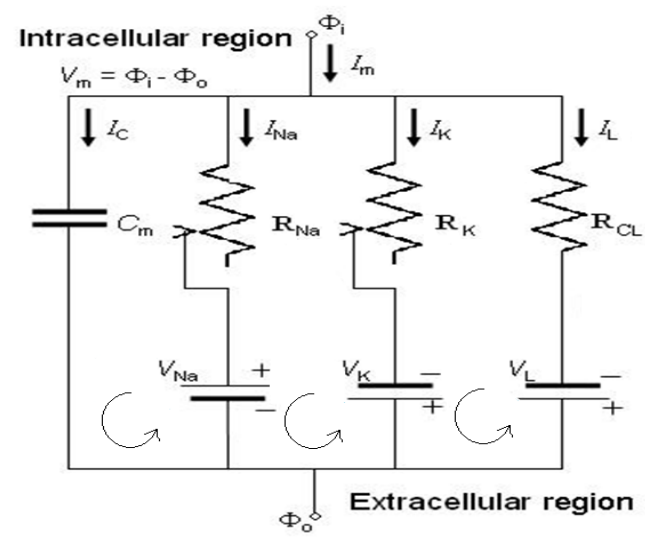

Fig. 1. Conduction through neural membrane

The first Kirchhoff law for the circuit can be written:

$$
C_{m} \frac{d V}{d t}=-g_{N a}\left(V-V_{N a}\right)-g_{K}\left(V-V_{K}\right)-g_{L}\left(V-V_{L}\right)+I_{m}
$$

If the potential is not spatially constant, what is usually happening, the equation should contain an additional Laplacian term. This term will be neglected here, but will be considered in the next section of the paper. Here we intend to show a simple way on how the complicated Hodgkin-Huxley model can be reduced to a binary one of the Hopfield type. Such a reduction supposes to impose supplementary constraints on the dynamics or more precisely on the time evolutions of the voltages on capacity, respectively on resistances. The idea is to consider that the voltage dependence of $g_{N a}$ and $g_{K}$ determines a delay on which the voltage $U$ on the resistive channels will go to the voltage $V$ on the capacitor. So, we make a distinction between the voltage $V$ expressing the capacitive properties of the cell and the voltage $U$ reflecting the membrane conductance. The two dynamical variables have quite different time scale evolutions, but tend asymptotically to each other. The equation (1) can be spited in two first order differential equations of the general form:

$$
\begin{aligned}
& C_{m} \frac{d V}{d t}=-f(V, U, t)+I_{m}(t) \\
& \frac{d U}{d t}=g(V, U, t)
\end{aligned}
$$

The requirement that $U$ approach asymptotically $V$ imposes that $g(V, U) \stackrel{U \rightarrow V}{\longrightarrow} 0$.

The first equation in (2) can be solved assuming small values of the membrane capacitance. This assumption is in agreement with the experimental data and with the fact 
that the membrane potential $V$ tends to remain constant almost the time. Under this assumption, we have:

$$
C_{m} \frac{d V}{d t} \approx 0 \Rightarrow f(V, U, t) \approx I_{m}(t)
$$

The equation (3) can be solved and it gives an expression of the voltage $V$ in terms of $U$ for various constant values of the external currents $I_{m}$.

It has been noted [2] that $V$ is a multi-valued function of $U$. More precisely, the representation $V=f(U)$ can be mainly splitted in two branches: a mostly liner branch for voltages under the resting potentials $V=U=-65 \mathrm{mV}$ and a second nonlinear one for higher values. To separate the two branches a binary variable $S$ can be used. It can be chosen so that $S=+1$ for the upper region and $S=-1$ for the other one. The dependence $V=f(U)$ becomes now a function of the form $V=f(U, S)$ and so the second equation from (2). Simulations have shown that the external currents $I_{m}$ have not an appreciable influence on the dynamics in the region $S=+1$ and a good agreement with the real neuron behavior can be described replacing the system (2) by the following equations:

$$
\frac{d U}{d t}=a-b \cdot U(t)+c(1-S) I_{m}(t)
$$

The coefficients $a, b$ and $c$ are numerical coefficients, depending on the characteristics of the neuron. A voltage shift with the resting potential and a current re-scaling allow to partially absorb such irrelevant coefficients. We will consider:

$$
U(t)-U_{0} \rightarrow u(t) ; c I_{m}(t) \rightarrow v(t)
$$

With the notation $A=a-b U_{0}$, the equation (4) becomes:

$$
\dot{u}(t)=-b \cdot u(t)+A+(1-S) v(t)
$$

The change of variables (5) leads to an expression for the binary variable of the type:

$$
S=\operatorname{sign}\left[S+\frac{30}{C}-u+(1-S) v\right]
$$


The equations (6) and (7) represent direct route to the Hopfield model for neural networks. This model is given by the following equation:

$$
\dot{u}_{k}(t)=-b_{k} u_{k}+\sum_{j=1}^{n} w_{j k} G_{j}\left(u_{j}\right)+v_{k}(t) \quad k=1, \ldots, n
$$

The connection becomes evident if we are considering $n$ neurons interconnected with the interaction weights:

$$
w_{j k}=\begin{array}{ll}
w_{k j} & j \neq k \\
0 & j=k
\end{array} \quad j, k=1, \ldots, n
$$

The functions $G_{j}\left(u_{j}\right)$ describe the contribution of the neuron $j$ to the impulse traveling through the neuron $k$. It is clear that for one single neuron, the condition (9) assures the reduction of the equation (8) to the model (6).

As we already mentioned, the Hopfield model (8) has been intensively studied, both as a model for a real neural network and as a mathematical tool for solving optimization problems. We will limit here to mention some results concerning the stability of the model, one of its strong characteristics. In [4], the stability around the equilibrium point $\dot{u}_{k}=0$ has been studied, in the absence of external inputs, $v_{k}=0$ and for the choice of the transfer function in the form:

$$
G_{k}\left(u_{k}\right)=a_{k} \cdot \arctan \left(\lambda_{k} u_{k}\right) \quad k=1, \ldots, n
$$

In some cases the external currents cannot be considered as vanishing, but in almost all neural network applications they can be considered constants over a time interval of interest, $v_{k} \equiv c_{k}$. Within this last assumption, the equilibrium points are defined as solution of the following equation:

$$
-b_{k} u_{k}^{*}+\sum_{j=1}^{n} w_{j k} G_{j}\left(u_{j}^{*}\right)+c_{k}=0
$$

Here are the main results reported in [4]. If some $a_{k}>0$ and $\lambda_{k}>0$ exist, the unperturbed system accepts a Lyapunov function of the form:

$$
L(u)=\frac{1}{2} \sum_{k=1}^{n} \alpha_{k} u_{k}^{2}
$$


The point $u=0$ is an equilibrium point of the neural network (8). It is exponentially stable if:

1. all the external inputs are zero: $v_{k}(t)=0, k=1, \ldots, n$

2. the interconnections satisfy the estimate: $u_{k} w_{k j} G_{j}\left(u_{j}\right) \leq\left|u_{k}\right| \cdot N_{j k}\left|u_{j}\right|$, with $N_{j k}$ real constants.

3. there exists a vector $\alpha \in R_{+}^{N}$, such that the test matrix $\mathrm{S}=\left[\mathrm{s}_{\mathrm{ij}}\right]$ is negative definite, where

$$
s_{i j}= \begin{cases}\alpha_{i}\left(-b_{i}+a_{i i}\right) & i=j \\ \frac{1}{2}\left(\alpha_{i} a_{i j}+\alpha_{j} a_{j i}\right. & i \neq j\end{cases}
$$

Consider now the Hopfield model in the perturbed version:

$$
\dot{u}_{k}=-\widetilde{b}_{k} u_{k}+\sum_{j=1}^{n} \widetilde{A}_{j k} \widetilde{G}_{j}\left(u_{j}\right)+v_{k}(t)
$$

where

$$
\begin{gathered}
\bar{b}_{k}=b_{k}+\Delta b_{k} ; \quad \bar{A}_{j k}=A_{j k}+\Delta \bar{A}_{j k} \\
\widetilde{G}_{j}\left(u_{j}\right)=G_{j}\left(u_{j}\right)+\Delta \widetilde{G}_{j}\left(u_{j}\right)\left(\Delta \widetilde{G}_{j}\left(u_{j}\right) \in C^{2}[\Re,(-1,1]), \quad 1 \leq j \leq n\right.
\end{gathered}
$$

The main system (8) is "robust" if for every its asymptotically stable equilibrium $u_{e}$ and for every $\varepsilon>0$, there is $\delta>0$ so that, when $\max \left\{\Delta b_{i}|,| \Delta A_{i j}|,| \Delta G_{j}\left(u_{e}\right),\left|\Delta G_{j}^{\prime}\left(u_{e}\right),\right| U_{i} \mid\right\}<\delta$, the perturbed system (14) has an equilibrium $\tilde{u}_{e}$ with

$$
\left|\widetilde{u}_{e}-u_{e}\right|<\varepsilon
$$

\section{Neural networks with inhomogeneous potentials}

In the previous section the case of a constant potential along the axon has been considered. As we already mentioned, this is a strong assumption which is usually not observed by real neurons. A more realistic supposition is that the flow travel with constant velocity $\Theta$ and that the impulse maintains its original form during the propagation. Under these conditions, the voltage depends on the point where is calculated, $u=u(x, t)$, the dynamics obeys the wave equation and the propagation regime is called steady-state regime. It is described by an equation of the form:

$$
\frac{\partial^{2} u}{\partial x^{2}}=\frac{1}{\Theta^{2}} \frac{\partial^{2} u}{\partial^{2} t}
$$


Let us come back now to the Hodgkin-Huxley model for a long squid axon. Let us denote by $r_{m}$ the electric resistance of the unit length of the axon and suppose that the variation of the potential along the axon satisfies a relation of the form:

$$
\frac{\partial u}{\partial x}=I_{m} r_{m}
$$

In the absence of ion pumps, the current propagating along the axon is a sum of the intracellular current and extracellular currents (see Fig.1). The previous relation becomes:

$$
\frac{\partial u}{\partial x}=I_{m} r_{m}=I_{i} r_{i}+I_{0} r_{0}
$$

Taking a new derivation of the previous relation we get:

$$
\frac{\partial^{2} u}{\partial x^{2}}=r_{i} \frac{\partial I_{i}}{\partial x}+r_{0} \frac{\partial I_{0}}{\partial x}
$$

The conservation of total charge imposes that: $\frac{\partial I_{i}}{\partial x}=\frac{\partial I_{0}}{\partial x}=v_{m}$, so the previous relation becomes:

$$
\frac{\partial^{2} V}{\partial x^{2}}=\left(r_{i}+r_{0}\right) v_{m}
$$

The equation from above is called general cable equation. Substituting this last equation in equation (17), we obtain:

$$
v_{m}=\frac{1}{\Theta^{2}\left(r_{i}+r_{0}\right)} \frac{\partial^{2} u}{\partial t^{2}}
$$

This expression can be considered into the Hopfield model (6) and, passing to the "wave" coordinate $\tau=t-\frac{x}{c}$, we get second order differential equations of the form:

$$
\ddot{\mathrm{u}}(\tau)-\mathrm{A} \cdot \dot{\mathrm{u}}(\tau)-\mathrm{B} \cdot \mathrm{u}=0
$$

Where the coefficients $A$ and $B$ can be simple identified and $\dot{u} \equiv \frac{d u}{d \tau} ; \ddot{u} \equiv \frac{d^{2} u}{d \tau^{2}}$. We studied numerically the behavior of the voltage along the wave, and the results are presented in the figure below. It is interesting that the flow can expand for small values of $A$ and strongly damp for bigger values. 


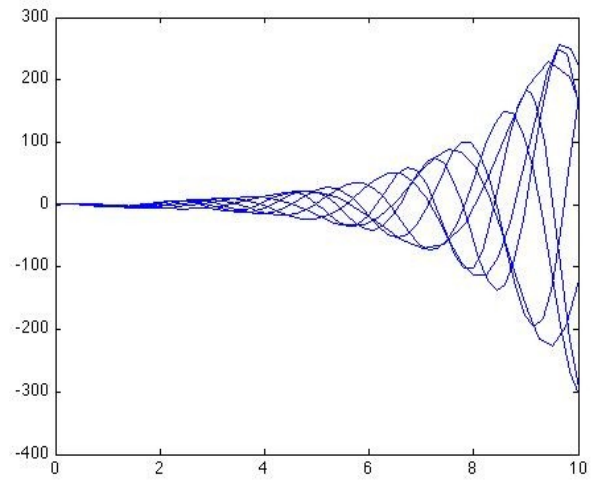

$A=1 ; B=-1, . .,-4$

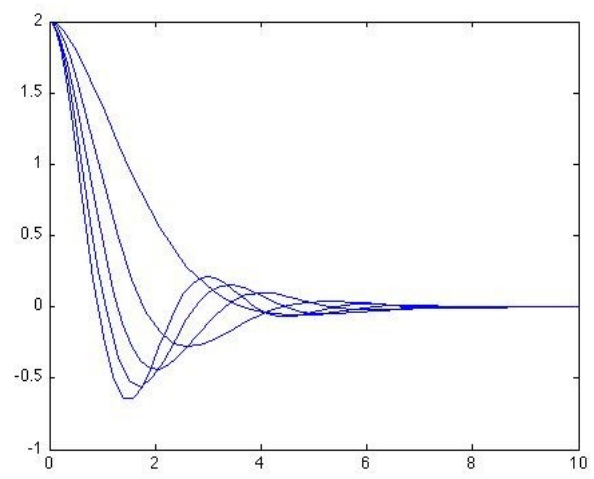

$A=-1.5 ; B=-1, . .,-100$

Fig. 2. Numerical analyses

In the considered situation, when the cable equation is valid for the axon, the neural flow propagates as damped oscillations. The equation is similar with what Van der Pol equation gives, mixing chaotic behavior with regular orbits.

\section{Conclusions}

The description of the transport phenomena along and across neurons is a not yet elucidated problem. There are many models trying to explain these phenomena, starting from very complex ones as Hodgkin-Huxley, continuing with integrate or fire models, till very simple ones as binary Hopfield-type models. The reduction of the complexity of a model allows detailed studies on network characteristics but spoils its biological content. Hopfield model for neural networks represents a special situation. Its simplicity gave force to the model and allowed to explain how the neural impulse is transmitted from one neuron to another. Despite the fact that it is designed to describe a network, the model works for individual neurons, too. It contains the main ingredients of Hodgkin-Huxley model and explains the stability of the flow along the axon. We presented here the main results concerning the stability of an isolated neuron, both for the case when a homogeneous voltage is considered and for the case of the propagation with a constant velocity. Forthcoming investigation will be devoted to a more carefully reduction of the model using the similarity reduction procedure [8]. Another possible approach which will be considered will be based not on the reduction, but on the description of the neuron using an extended phase space, as in [9]. These approaches will be complemented by trying to implement electronic circuits to model the neural flow. 


\section{Acknowledgments}

This work was partially supported by the strategic grant POSDRU/CPP107/DMI1.5/S/78421, Project ID 78421 (2010), co-financed by the European Social Fund - Investing in People, within the Sectorial Operational Programme Human Resources Development 2007 - 2013.

\section{References}

[1]J. J. Hopfield, Proc. Nat Acad. Sci. USA 79 (1982), 2554-2558.

[2]L F Abbott, J. Phys. A: Math. Gen. 23 (1990), 3835-3860.

[3]M. Kouh, T.Poggio, Neural Comput. 20 (2008) 1427-1451.

[4]M.Condon, G.G. Grahovski, in Scientific Computing in Electrical Engineering SCEE $2008,579-586$

[5]Wen, U.-P. et al., European Journal of Operational Research (2008), doi:10.1016/j.ejor.2008.11.002

[6]L. Hodgkin, A. F. Huxley, J. Physiol 119 (1952), 500-544

[7]R,.Constantinescu, C.Ionescu, M.Stoicescu - Rom.J.Phys, Vol. 58 (2013), no. 5-6.

[8]R.Cimpoiasu, R.Constantinescu, J. Nonlin. Math. Phys. 13 (2006), 285-292.

[9]R.Constantinescu, J.Math.Phys. Vol. 38 (1997) 2786-2794. 